\title{
DECOMPOSITIONS OF SPACES DETERMINED BY COMPACT SUBSETS
}

\author{
YOSHIO TANAKA
}

\begin{abstract}
Let $X$ be a $k^{\prime}$-space, and let $₹$ be a closed cover of (locally) compact subsets of $X$. Then $X$ is decomposed into a closed discrete subset and a locally compact subset if $X$ is dominated by $\mathcal{F}$, or $X$ has the weak topology with respect to a point-countable cover $\mathcal{F}$. Here, a cover of a space is point-countable if every point is in at most countably many elements of the cover.
\end{abstract}

Introduction. We assume that all spaces are Hausdorff, and that all maps are continuous and onto. Suppose that $f: X \rightarrow Y$ is a closed map. When $X$ is a locally compact paracompact space, $\mathrm{K}$. Morita [14] showed that $Y$ is decomposed into a closed discrete subset and a locally compact subset. When $X$ is a metric space, N. Lašnev [9] showed that $Y$ is decomposed into a $\sigma$-discrete subset and a metric subset. Here, a subset of $Y$ is $\sigma$-discrete if it is a countable union of closed discrete subsets of $Y$. However, if $f$ is a quotient map, not every paracompact space $Y$ is decomposed into a $\sigma$-discrete subset and a subset which is metric or locally compact, even if every $f^{-1}(y)$ is finite and $X$ is locally compact separable metric; see Example 3.1.

Now, in terms of weak topology, let us recall some definitions related to quotient spaces of locally compact spaces. Let $C$ be a cover of a space $Z$. Then $Z$ is determined by $C$ [8], or $Z$ has the weak topology with respect to $C$, if $F \subset Z$ is closed in $Z$ if and only if $F \cap C$ is relatively closed in $C$ for every $C \in C$. Here we can replace "closed" by "open". A space is a $k$-space if it is determined by the cover of all compact subsets. It is well known that every $k$-space is characterized as a quotient image of a locally compact (paracompact) space. Let $₹$ be a closed cover of a space $Z$. Then $Z$ is dominated by $\mathcal{F}[\mathbf{1 0}]$, if the union of any subcollection $\mathcal{F}^{\prime}$ of $\mathcal{F}$ is closed in $Z$ and the union is determined by $\mathcal{F}^{\prime}$. Every CW-complex is dominated by compact metric subsets [19]. Every locally compact paracompact space $X$, as well as every closed image of $X$, is dominated by a hereditarily closure-preserving cover of compact subsets. Here, a cover $\left\{C_{\alpha}\right\}$ of a space is hereditarily closure-preserving if $\overline{\bigcup_{\alpha} B_{\alpha}}=\bigcup_{\alpha} \overline{B_{\alpha}}$ for any $B_{\alpha} \subset C_{\alpha}$. A space $Y$ is a $k^{\prime}$-space [3], if whenever $y \in \bar{A}$, then $y \in \overline{A \cap C}$ for some compact subset $C$ of $Y$. A space $Y$ is Fréchet, if whenever $y \in \bar{A}$, there exists a sequence in $A$ converging to $y$. Every locally compact and every Fréchet space is a $k^{\prime}$-space. Every $k^{\prime}$-space is characterized as an image of a locally compact (paracompact) space under a pseudo-open map [2]. Recall that a map $f: X \rightarrow Y$ is pseudo-open [2], or hereditarily quotient, if for any

Received by the editors March 15, 1985 and, in revised form, June 28, 1985.

1980 Mathematics Subject Classification. Primary 54B15, 54D45; Secondary 54C10, 54D50, $54 \mathrm{E} 45$.

Key words and phrases. Weak topology, $k$-space, $k^{\prime}$-space, locally compact space, quotient map, pseudo-open map, closed map. 
$y \in Y$ and any open subset $U$ containing $f^{-1}(y), y \in \operatorname{int} f(U)$; equivalently, for any $A \subset Y, f \mid f^{-1}(A)$ is quotient [2]. Closed maps and open maps are psuedo-open, and pseudo-open maps are quotient.

In this paper, we show that every $k^{\prime}$-space (more generally, singly bi- $k$-space [12]) dominated by locally compact subsets, or determined by a point-countable closed cover of locally compact subsets, is decomposed into a closed discrete subset and a locally compact subset. Thus, for a $k^{\prime}$-space $Y$, if $Y$ is a $C W$-complex or an image of a locally compact paracompact space under a quotient map with each point-inverse Lindelöf, then $Y$ is decomposed into a closed discrete subset and a locally compact subset.

1. Spaces determined by a point-countable cover of compact subsets. Let $Y$ be a space. For a cover $C$ of $Y$, let us consider the following condition (*) with respect to $C$ :

(*) If $y \in \bar{A}$, then $y \in \overline{A \cap C}$ with $y \in C$ for some $C \in C$.

LEMMA 1.1. Let $Y$ be a $k$-space, and $C$ be a point-countable cover of $Y$. If $Y$ satisfies (*) with respect to $C$, then $Y_{0}=\left\{y \in Y ; y \notin\right.$ int $\bigcup C^{\prime}$ for any finite $\left.C^{\prime} \subset C\right\}$ is discrete in $Y$.

Proof. Suppose that $Y_{0}$ is not discrete in $Y$. Then some $A \subset Y_{0}$ is not closed in $Y$. Since $Y$ is a $k$-space, there exists a compact subset $K$ such that $K \cap A$ is not closed. Since $K \cap A$ is an infinite subset of $K$, there exists an infinite subset $\left\{y_{n} ; n \in N\right\}$ of $K \cap A$ accumulating at some $y \in Y$ with $y_{n} \neq y$. Let $V_{n}$ be a neighborhood of $y_{n}$ with $\overline{V_{n}} \not \supset y$. Let $\{C \in C ; C \ni y\}=\left\{C_{1}, C_{2}, \ldots\right\}$. For each $n \in N$, let $B_{n}=\bigcup_{m \leq n} C_{m}$, and let $A_{n}=V_{n}-B_{n}$. Since each $y_{n} \in Y_{0}, y_{n} \in \overline{A_{n}}$. Thus, $y \in \overline{\bigcup_{n \in N} A_{n}}$. By condition $(*), y \in \overline{\left(\bigcup_{n \in N} A_{n}\right) \cap C_{i}}$ for some $i \in N$. Thus, $y \in \overline{A_{j}}$ for some $j<i$, hence $y \in \overline{V_{j}}$. This contradiction completes the proof of the lemma.

According to E. Michael [12], a space is singly bi-k, if it is a pseudo-open image of a paracompact $M$-space. Here, a space is paracompact $M$-space if it admits a perfect map onto a metric space. $k^{\prime}$-spaces are singly bi- $k$, and singly bi-k-spaces are $k$-spaces [12].

LEMMA 1.2. If $Y$ is a singly bi-k-space determined by a point-countable closed cover $C$, then $Y$ satisfies (*) with respect to $C$.

Proof. Let $y \in \bar{A}$. Since $Y$ is singly bi- $k$, by [12, Definition 5.E.1], there exists a sequence $\left\{A_{n} ; n \in N\right\}$ such that $y \in \overline{A \cap A_{n}}$ for all $n$, and if $y_{n} \in A_{n}$, then $\left\{y_{n} ; n \in N\right\}$ has an accumulation point in $Y$. Thus, as in the proof of $[\mathbf{1 7}$, Lemma 6], some $A_{n}$ is covered by a finite subcollection $C^{\prime}$ of $C$. Then $y \in \overline{A \cap C}$ with $y \in C$ for some $C \in C^{\prime}$. Then $Y$ satisfies $(*)$ with respect to $C$.

From Lemmas 1.1 and 1.2, we have

THEOREM 1.3. If $Y$ is a singly bi-k-space determined by a point-countable closed cover of locally compact subsets, then $Y$ is decomposed into a closed discrete subset and a locally compact subset.

Example 3.1 shows that the singly bi- $k$-ness of $Y$ is essential in Theorem 1.3, and also in Theorem 1.4 below.

Recall that a space is $\sigma$-metric if it is a countable union of closed metric subsets. 
THEOREM 1.4. Let $Y$ be a singly bi-k-space determined by a point-countable closed cover $\left\{Y_{\alpha}\right\}$ of metric subsets. Then (1) and (2) below hold.

(1) $Y$ is decomposed into a closed discrete subset and a subset with a pointcountable base.

(2) If $Y$ is a paracompact space, or a regular space with each $Y_{\alpha}$ locally separable, then $Y$ is a $\sigma$-metric space decomposed into a closed discrete subset and a metric subset.

ProOF. (1) By Lemmas 1.1 and 1.2, $Y$ is decomposed into a closed discrete subset and a locally metric subset $Y^{\prime}$ of $Y$. Since $Y^{\prime}$ is open in $Y, Y^{\prime}$ is determined by a point-countable closed cover $\left\{Y_{\alpha} \cap Y^{\prime}\right\}$ of $Y^{\prime}$. Let $X=\sum_{\alpha}\left(Y_{\alpha} \cap Y^{\prime}\right)$, where $\sum$ denotes topological sum. Then $X$ is metric, and the obvious map of $X$ onto $Y^{\prime}$ is a quotient and $s$-map (i.e., each point-inverse is separable). Since $Y^{\prime}$ is first countable, by $\left[6\right.$, Theorem $\left.1^{\prime}\right] Y^{\prime}$ has a point-countable base.

(2) For $y \in Y$, let $A=\bigcup\left\{Y_{\alpha} ; Y_{\alpha} \ni y\right\}$. Since $Y$ is singly bi-k, by Lemma 1.2, $y \notin \overline{X-A}$, hence $y \in \operatorname{int} A$. Thus $Y$ is a locally $\sigma$-metric space. In case where $Y$ is paracompact, $Y$ is a $\sigma$-metric space. Then the open subset $Y^{\prime}$ in (1) is an $F_{\sigma}$-set of $Y$. Hence $Y^{\prime}$ is paracompact. Since $Y^{\prime}$ is locally metric, $Y^{\prime}$ is metric. In case where $Y$ is regular and each $Y_{\alpha}$ is locally separable, each $Y_{\alpha}$ is determined by a locally finite closed cover $\left\{Y_{\alpha \beta} ; \beta\right\}$ of separable metric subsets. Hence $Y$ is determined by a point-countable closed cover $\left\{Y_{\alpha \beta} ; \alpha, \beta\right\}$ of separable metric subsets. But $Y$ is singly bi-k, hence is Fréchet by Lemma 1.2. Then, since $Y$ is regular, $Y$ is paracompact by [8, Corollary 8.9]. As seen above, then, $Y$ is $\sigma$-metric and $Y_{1}$ is metric. Therefore, in any case, $Y$ is a $\sigma$-metric space decomposed into a closed discrete subset and a metric subset.

COROLlaRY 1.5. Let $f: X \rightarrow Y$ be a quotient s-map such that $X$ is locally compact metric. If $Y$ is a regular $k^{\prime}$-space, then $Y$ is a $\sigma$-metric space decomposed into a closed discrete subset and a (locally compact) metric subset.

ProOF. Since $X$ is determined by a locally finite cover $\left\{X_{\alpha}\right\}$ of compact metric subsets, $Y$ is determined by a point-countable cover $\left\{f\left(X_{\alpha}\right)\right\}$ of compact metric subsets. Thus the corollary follows from Theorem 1.4(2).

The local compactness of $X$ in Corollary 1.5 is essential. Indeed, there exists a closed image $Y$ of a separable metric space such that $Y$ is not $\sigma$-metric [7], hence is not decomposed into a closed discrete subset and a metric subset. Also, there exists a paracompact space $X$ which is an open $s$-image of a metric space such that $X$ is not a $\sigma$-space (hence, not $\sigma$-metric), nor is $X$ decomposed into a $\sigma$-discrete subset and a metric subset; see Example 3.2.

Recall that a space $X$ is meta-Lindelöf if every open cover of $X$ has a pointcountable open refinement. Every meta-compact space is meta-Lindelöf.

LEMMA 1.6. Let $f: X \rightarrow Y$ be a quotient map with $X$ locally compact. Suppose that (a) or (b) below holds.

(a) $X$ is meta-Lindelöf and every $f^{-1}(y)$ is separable.

(b) $X$ is paracompact and every $f^{-1}(y)$ is Lindelöf.

If $Y$ is a singly bi-k-space; equivalently, $f$ is pseudo-open, then $Y$ is determined by a point-countable cover $C$ such that each element of $C$ has a compact closure, and $Y$ satisfies $(*)$ with respect to $C$. 
ProOF. Let us assume case (a) (the proof for case (b) is similar). Then $X$ is determined by a point-countable open cover $\left\{G_{\alpha}\right\}$ such that $G_{\alpha}$ are compact. Since $f$ is a quotient s-map, $Y$ is determined by a point-countable cover $C=\left\{f\left(G_{\alpha}\right)\right\}$. To show that $f$ is pseudo-open, let $y \in Y$ and $U$ be any open subset containing $f^{-1}(y)$. Suppose that $y \notin \operatorname{int} f(U)$, hence $y \in \overline{Y-f(U)}$. Since $Y$ is singly bi- $k$, as in the proof of Lemma 1.2 (cf. [17, Lemma 6]), $y \in \overline{(Y-f(U)) \cap f\left(G_{\alpha_{0}}\right)}$ for some $\alpha_{0}$. Thus $y \in \overline{K-f(U)}$, where $K=f\left(\overline{G_{\alpha_{0}}}\right)$. Let $g=f \mid \overline{G_{\alpha_{0}}}$. Since $\overline{G_{\alpha_{0}}}$ is compact, $g$ is closed. But $g^{-1}(y) \subset U \cap \overline{G_{\alpha_{0}}}$. Thus $y \in \operatorname{int}_{K} g\left(U \cap \overline{G_{\alpha_{0}}}\right)$, hence $y \notin \overline{K-f(U)}$. This is a contradiction. Thus $f$ is pseudo-open. To show that $Y$ satisfies $(*)$ with respect to $C$, let $y \in \bar{A}$. Since $f$ is pseudo-open, $f^{-1}(y) \cap \overline{f^{-1}(A)} \neq \varnothing$. Let $x \in$ $f^{-1}(y) \cap \overline{f^{-1}(A)}$, and let $x \in G_{\alpha_{1}}$. Then $x \in \overline{f^{-1}(A) \cap G_{\alpha_{1}}}$, thus $y \in \overline{A \cap f\left(G_{\alpha_{1}}\right)}$ and $y \in f\left(G_{\alpha_{1}}\right)$. Thus $Y$ satisfies $(*)$ with respect to $C$.

By Lemmas 1.1 and 1.6, we have the following theorem. Example 3.1 shows that "pseudo-open map" cannot be weakened to "quotient map", and Example 3.3 shows that the condition of $f^{-1}(y)$ is essential in the theorem, even if $X$ is locally compact metric.

THEOREM 1.7. Let $f: X \rightarrow Y$ be a pseudo-open map, or a quotient map with $Y$ a $k^{\prime}$-space. If $X$ is a locally compact space, and (a) or (b) of Lemma 1.6 holds, then $Y$ is decomposed into a closed discrete subset and a locally compact subset.

\section{Spaces dominated by compact subsets.}

LEMMA 2.1. Let $X$ be dominated by a closed cover $\left\{X_{\alpha}\right\}$, and let $Y_{\alpha}=$ $\overline{X_{\alpha}-\bigcup_{\beta<\alpha} X_{\beta}}$. If $X$ is singly bi-k, then $\left\{Y_{\alpha}\right\}$ is a hereditarily closure-preserving closed cover of $X$.

Proof. Suppose that $\left\{Y_{\alpha}\right\}$ is not hereditarily closure-preserving. Then there exist closed subsets $A_{\alpha}$ of $Y_{\alpha}$ such that $\bigcup_{\alpha} A_{\alpha}$ is not closed in $X$. Since $X$ is a $k$ space, there exists a compact subset $K$ of $X$ such that $\left(\bigcup_{\alpha} A_{\alpha}\right) \cap K$ is not closed in $K$. Then there exists an infinite subset $\left\{x_{n} ; n \in N\right\}$ of $X$ such that $x_{n} \in A_{\alpha_{n}} \cap K$ with $\alpha_{n}<\alpha_{n+1}$. Let $x \in X$ be an accumulation point of $\left\{x_{n} ; n \in N\right\}$, which we may suppose different from all $x_{n}$. Let each $V_{n}$ be a neighborhood of $x_{n}$ with $\overline{V_{n}} \not \supset x$. Let $B_{n}=V_{n} \cap\left(X_{\alpha_{n}}-\bigcup_{\beta<\alpha_{n}} X_{\beta}\right), B=\bigcup_{n \in N} B_{n}$, and let $C=\bigcup_{n \in N} X_{\alpha_{n}}$. Then $x \in \bar{B}^{C}$. But $C$ is a singly bi-k-space determined by a countable closed cover $\left\{X_{\alpha_{n}} ; n \in N\right\}$. Thus, by Lemma $1.2, x \in \overline{B \cap X_{\alpha_{i}}}$ for some $i \in N$. Hence $x \in \overline{B_{j}}$ for some $j \leq i$, thus $x \in \overline{V_{j}}$. This contradiction completes the proof of the lemma.

Since every $k^{\prime}$-space is singly bi- $k$, the "only if" part of the following follows from Lemma 2.1. The "if" part is easy.

COROLlARY 2.2. Let $X$ be dominated by a cover $\left\{X_{\alpha}\right\}$ of compact subsets, and let $Y_{\alpha}=\overline{X_{\alpha}-\bigcup_{\beta<\alpha} X_{\beta}}$. Then $X$ is a $k^{\prime}$-space if and only if $\left\{Y_{\alpha}\right\}$ is hereditarily closure-preserving.

LEMMA 2.3. Let $\mathcal{F}$ be a hereditarily closure-preserving closed cover of a space $Y$. If $Y$ is a $k$-space, then $Y_{0}=\{y \in Y ; \mathcal{F}$ is not locally finite at $y\}$ is discrete in $Y$.

Proof. Suppose that $Y_{0}$ is not discrete in $Y$. Then some $A \subset Y_{0}$ is not closed in $Y$. Since $Y$ is a $k$-space, there exists a compact subset $K$ such that 
$K \cap A$ is not closed. Thus there exists an infinite subset $\left\{y_{n} ; n \in N\right\}$ of $K \cap A$ accumulating at some $y \in Y$. Since $y_{n} \in Y_{0}, \mathcal{F}$ is not point-finite at $y_{n}$, so there exists $\left\{F_{n} ; n \in N\right\} \subset \mathcal{F}$ with $y_{n} \in F_{n}$. Thus $\left\{y_{n} ; n \in N\right\}$ is discrete in $Y$, a contradiction.

THEOREM 2.4. Let $Y$ be a singly bi-k-space dominated by a closed cover of locally compact subsets (resp. metric subsets). Then $Y$ is decomposed into a closed discrete subset and a locally compact subset (resp. metric subset).

PROOF. This theorem follows from Lemmas 2.1 and 2.3. For the parenthetic part, use the well-known fact that every space dominated by metric subsets is hereditarily paracompact [10 or $\mathbf{1 3}]$.

In the following corollary, the $k^{\prime}$-ness of $Y$ is essential; see Example 3.4.

COROLlarY 2.5. Let $Y$ be a $C W$-complex. If $Y$ is a $k^{\prime}$-space, then $Y$ is decomposed into a closed discrete subset and a locally compact metric subset.

THEOREM 2.6. The following are equivalent:

(1) $Y$ is a pseudo-open image of a paracompact $M$-space (or a locally compact space), and $Y$ is dominated by compact subsets.

(2) $Y$ is a closed image of a locally compact paracompact space.

Proof. $(2) \rightarrow(1)$ is straightforward. (1) $\rightarrow$ (2) follows from Lemma 2.1 and the fact that, for every hereditarily closure-preserving closed cover $\left\{Y_{\alpha}\right\}$ of $Y$, the obvious map of $\sum_{\alpha} Y_{\alpha}$ onto $Y$ is closed.

THEOREM 2.7. The following are equivalent:

(1) $Y$ is a pseudo-open image of a locally compact Lindelö space.

(2) $Y$ is a closed image of a locally compact Lindelöf space.

ProOF. It suffices to prove $(1) \rightarrow(2)$. Since $Y$ is a quotient image of a locally compact Lindelöf space, by [15], $Y$ is determined by a countable cover $C$ of compact subsets. Here we can assume that $C$ is increasing, so $Y$ is dominated by $C$, while, $Y$ is a $k^{\prime}$-space. Then (1) $\rightarrow(2)$ can be given using Lemma 2.1 .

3. Examples. A space $Y$ is a $q$-space $[\mathbf{1 1}]$ if each point of $Y$ has a sequence $\left\{U_{n} ; n \in N\right\}$ of open neighborhoods such that, if $y_{n} \in U_{n}$, then $\left\{y_{n} ; n \in N\right\}$ has an accumulation point in $Y$. First countable spaces and locally compact spaces, more generally spaces of pointwise-countable type [3], are $q$-spaces.

EXAMPLE 3.1. A regular Lindelöf space $Y$ is determined by a point-finite, countable cover $\left\{Z_{n} ; n \in \omega\right\}$ of compact metric subsets, hence $Y$ is a quotient finite-to-one image of a locally compact separable metric space $\sum_{n \in \omega} Z_{n}$. But, for any $\sigma$-discrete subset $Y_{0}$ of $Y, Y-Y_{0}$ is not a $q$-space.

PROOF. For each $n \in \omega$, define a subspace $Z_{n}$ of $\mathbf{R}^{3}$ by $Z_{0}=\{0\} \times A$, and $Z_{n}=A \times\{1 / n\}$, where $A=[0,1] \times[0,1]$. Let $Y=\bigcup_{n \in \omega} Z_{n}$, and let $U \subset Y$ be open in $Y$ whenever every $U \cap Z_{n}$ is open in $Z_{n}$. Then $Y$ is a space determined by a point-finite, countable cover $\left\{Z_{n} ; n \in \omega\right\}$ of compact metric subsets. For any $\sigma$-discrtete subset $Y_{0}$ of $Y$, let $Y_{1}=Y-Y_{0}$. Since each $Z_{n} \cap Y_{0}$ is countable (= at most countable), there exists $y_{0}=(0, t, 0) \in Y_{1}$ such that $(0, t, 1 / n) \in Y_{1}$ for all $n \in N$. But no sequences $\left\{z_{n} ; n \in N\right\}$ with $z_{n} \in Z_{n}-Z_{0}$ have accumulation points in $Y$. Thus $Y_{1}$ is not a $q$-space. 
EXAMPLE 3.2. A regular Lindelöf space $X$ which is an open $s$-image of a metric space, but for any $\sigma$-discrete subset $X_{0}$ of $X, X-X_{0}$ is neither a $\sigma$-space nor a $p$-space in the sense of A. V. Arhangel'skir [1]

ProOF. In view of the proof of [4, Problem 285, p. 146], there exists a subset $A$ of $[0,1]$ such that $A$ and its complement have size $2^{\omega}$, and $A$ does not contain $C-D$ for any uncountable compact subset $C$ of $[0,1]$ and for any countable subset $D$ of $[0,1]$. Let $X$ be the space obtained from $[0,1]$ by isolating the points of $A$. Since $X$ is a regular space with a point-countable base, $X$ is an open $s$-image of a metric space by [16]. Now, let $X_{0}$ be any $\sigma$-discrete subset of $X$, and let $X_{1}=X-X_{0}$. Since $X$ is Lindelöf, $X_{0}$ is countable. Thus $X_{1}$ is not separable. But $X_{1}$ is Lindelöf. Hence $X_{1}$ is not a $\sigma$-space. Also, $X_{1}$ is not a $p$-space, for every paracompact $p$-space with a point-countable base is metric [5].

EXAMPLE 3.3. A regular Lindelöf space $Y$ which is a pseudo-open image of a locally compact metric space, but for any $\sigma$-discrete subset $Y_{0}$ of $Y, Y-Y_{0}$ is neither first countable nor a $p$-space.

ProOF. Let $T=D \cup\{\infty\}$ be the one point compactification of an uncountable discrete space $D$. Let $Z=T \times T$. Suppose that $Z$ is decomposed into a $\sigma$-discrete subset $Z_{0}$ and a subset $Z_{1}$ of $Z$. Since $Z_{0}$ is countable, there exists $d \in D$ such that $(\infty, d) \in Z_{1}$, but $Z_{1}$ has no countable bases at the point $(\infty, d)$. Then $Z_{1}$ is not first countable. Now, let $Y$ be a topological sum of $Z$ and the space $X$ of Example 3.2. Since $Y$ is Fréchet, $Y$ is a pseudo-open image of a locally compact metric space by [2]. However, for any $\sigma$-discrete subset $Y_{0}$ of $Y, Y-Y_{0}$ is neither first countable nor a $p$-space.

EXAMPLE 3.4. A countable CW-complex $X$ such that for any $\sigma$-discrete subset $X_{0}$ of $X, X-X_{0}$ is not a $q$-space.

PROOF. For each $n \in N$, let $L_{n}$ be a copy $\square A_{n} B_{n} C_{n} D_{n}$ of a rectangle $\square A B C D$. Let $X$ be the space obtained from $\sum_{n \in N} L_{n}$ by identifying all of segments $\overline{A_{n} B_{n}}$ to a segment. Then $X$ is a countable $\mathrm{CW}$-complex satisfying the desired property.

\section{REFERENCES}

1. A. V. Arhangel'skiĭ, On a class of spaces containing all metric and all locally bicompact spaces, Soviet Math. Dokl. 4 (1963), 1051-1055.

2. Some types of factor mappings, and the relations between classes of topological spaces, Soviet Math. Dokl. 4 (1963), 1726-1729.

3. , Bicompact sets and the topology of spaces, Trans. Moscow Math. Soc. 13 (1965), $1-62$.

4. A. V. Arkhangel'skiǐ and V. I. Ponomarev, Fundamentals of general topology, Reidel, Dordrecht, 1984.

5. V. V. Filippov, On feathered paracompacta, Soviet Math. Dokl. 9 (1968), 161-164.

6. __ On factor s-mappings, Soviet Math. Dokl. 9 (1968), 1055-1057.

7. B. Fitzpatric, Jr., Some topologically complete spaces, Topology Appl. 1 (1971), 101-103.

8. G. Gruenhage, E. Michael and Y. Tanaka, Spaces determined by point-countable covers, Pacific J. Math. 113 (1984), 303-332.

9. N. Lašnev, Continuous decompositions and closed mappings of metric spaces, Soviet Math. Dokl. 6 (1965), 1504-1506.

10. E. Michael, Continuous selections. I, Ann. of Math. (2) 63 (1956) 361-382.

11. __ A note on closed maps and compact sets, Israel J. Math. 3 (1964), 173-176.

12. __ A quintuple quotient quest, Topology Appl. 2 (1972), 91-138.

13. K. Morita, On spaces having the weak topology with respect to closed coverings, Proc. Japan Acad. 29 (1954), 537-543. 
14. __, On closed mappings, Proc. Japan Acad. 32 (1956), 539-543.

15. __ On decomposition spaces of locally compact spaces, Proc. Japan Acad. 32 (1956), 544-548.

16. V. I. Ponomarev, Axioms of countability and continuous mappings, Bull. Acad. Polon. Sci. Sér. Math. Astronom. Phys. 8 (1960), 127-134. (Russian)

17. Y. Tanaka, Point-countable $k$-systems and products of $k$-spaces, Pacific J. Math. 101 (1982), 199-208.

18. Y. Tanaka and Zhou Hao-xuan, Spaces dominated by metric subsets, Topology Proc. 9 (1984), 149-163.

19. J. H. C. Whitehead, Combinatorial homotopy. I, Bull. Amer. Math. Soc. 55 (1949), 213245.

Department of Mathematics, TOKyo Gakugei University, 4-1-1, NukuikitaMACHI, KOGANEI-SHI, TOKYO, JAPAN 